\title{
Strongly log-biconvex Functions and Applications
}

\author{
Muhammad Aslam Noor ${ }^{1, *}$, and Khalida Inayat Noor ${ }^{2}$ \\ ${ }^{1}$ Mathematics Department, COMSATS University Islamabad, Islamabad, Pakistan \\ e-mail: noormaslam@gmail.com \\ 2 Mathematics Department, COMSATS University Islamabad, Islamabad, Pakistan \\ e-mail: khalidan@gmail.com
}

\begin{abstract}
In this paper, we consider some new classes of log-biconvex functions. Several properties of the log-biconvex functions are studied. We also discuss their relations with convex functions. Several interesting results characterizing the log-biconvex functions are obtained. New parallelogram laws are obtained as applications of the strongly log-biconvex functions. Optimality conditions of differentiable strongly log-biconvex are characterized by a class of bivariational inequalities. Results obtained in this paper can be viewed as significant improvement of previously known results.
\end{abstract}

\section{Introduction}

Convex functions and convex sets have played an important and fundamental part in the development of various fields of pure and applied sciences. Convexity theory describes a broad spectrum of very interesting developments involving a link among various fields of mathematics, physics, economics and engineering sciences. In recent years, various extensions and generalizations of convex functions and convex sets have been considered and studied using innovative ideas and techniques. Noor and Noor [15, 16, 17, 19] introduced Biconvex sets and biconvex functions, which inspired a great interest. They proved that the differentiable biconvex functions are biconvex functions and the converse is also true under

Received: March 28, 2021; Accepted: May 4, 2021

2010 Mathematics Subject Classification: 49J40.

Keywords and phrases: biconvex functions, global minimum, log-biconvex functions, variational inequalities.

${ }^{*}$ Corresponding author

Copyright (C) 2021 Authors 
certain conditions. Noor et al. [15, 16, 19] proved that the minimum of the differentiable biconvex functions are characterized by bivariational inequalities. For the applications, numerical methods and other aspects of biconvex functions, see [15, 16, 17, 19] and the references therein. It is known that more accurate and inequalities can be obtained using the log-convex functions than the convex functions. It is known that log-convex functions are convex functions, but the converse is not true. For example. the function $e^{x}$ is a log-convex function, but not convex. Hypergeometric functions including Gamma and Beta functions are log-convex functions, which have important applications in several branches of pure and applied sciences. Recently Noor et al. [12, 13, 18, have considered an equivalent formulation of log-convex to discuss several new hidden aspects. In recent years, it have been shown that the exponentially convex functions have important applications in information theory, big data analysis, machine learning and statistic. See, for example, [1, 2, 3, 16, 20, 21, 23] and the references therein. The exponentially convex(concave) functions are closely related to the log-convex functions, the origin of exponentially convex functions can be traced back to Bernstein [3].

Recently, Noor et al. [12, 13, 18] considered the equivalent formulation of log-convex functions and proved that the log-convex functions have similar properties as the convex functions enjoy. Inspired and motivated by the ongoing research in this interesting, applicable and dynamic field, we consider the concept of strongly log-biconvex functions. We discuss the basic properties of the log-biconvex functions. It is has been shown that the log-biconvex(biconcave) have nice properties. Several new concepts of strongly log-biconvex functions have been introduced and investigated. The difference (sum) of the strongly log-biconvex function and affine strongly log-biconvex function is again a log-biconvex function. New parallelogram laws are obtained as applications of the strongly log-biconvex affine function, which can be viewed as a novel application. We show that the local minimum of the log-convex functions is the global minimum. The optimal conditions of the differentiable strongly log-biconvex functions can be characterized by a class of variational inequalities, which is itself an interesting outcome of our main results. The ideas and techniques of this paper may be 
a starting point for further research in these different areas of mathematical programming, machine learning and related optimization problems.

\section{Preliminary Results}

Let $K$ be a nonempty closed set in a real Hilbert space $H$. We denote by $\langle\cdot, \cdot\rangle$ and $\|\cdot\|$ be the inner product and norm, respectively. Let $F: K \rightarrow R$ be a continuous function.

Definition 1. [5] A set $K$ in $H$ is said to be convex set, if

$$
u+t(v-u) \in K, \quad \forall u, v \in K, t \in[0,1] .
$$

Definition 2. 5] A function $F$ is said to be convex, if

$$
F((1-t) u+t v) \leq(1-t) F(u)+t F(v), \quad \forall u, v \in K, \quad t \in[0,1] .
$$

Polyak 21] introduced the concept of strongly convex functions in optimization and mathematical programming.

Definition 3. A function $F$ is said to be a strongly convex, if there exists a constant $\mu \geq 0$ such that

$F((1-t) u+t v) \leq(1-t) F(u)+t F(v)-\mu t(1-t)\|v-u\|^{2}, \quad \forall u, v \in K, \quad t \in[0,1]$.

Clearly every strongly convex function is a convex function, but the converse is not true. For the applications of strongly convex functions in variational inequalities, differential equations and equilibrium problems, see [6, 7, 8 , 11, 12, 13, 14, 15, 16, 19, 20, 22, 24 and the references therein.

In many problems, the underlying set may not a convex set. To overcome this deficiency, Noor et al. [16, 17, 19] introduced the biconvex sets and biconvex functions with respect to an arbitrary bifunction, which can be viewed as important generalization of the convexity and inspired a great interest in nonlinear mathematical programming. 
Definition 4. [16, 17, 19] The set $K_{\beta}$ in $H$ is said to be biconvex set with respect to an arbitrary bifunction $\beta(.-$.), if

$$
u+t \beta(v-u) \in K, \quad \forall u, v \in K_{\beta}, t \in[0,1] .
$$

We would like to emphasize that, if $u+\beta(v-u)=v, \quad \forall u, v \in K_{\beta}$, then $\beta(v-u)=v-u$. Consequently, the biconvex set $K_{\beta}$ reduces to the convex set $K$. Thus, $K_{\beta} \subset K$. This implies that every convex set is a biconvex set, but the converse is not true.

Definition 5. A strictly positive function $F$ is said to be biconvex with respect to an arbitrary bifunction $\beta(.-$.), if

$$
F(u+t \beta(v-u)) \leq(1-t) F(u)+t F(v), \quad \forall u, v \in K_{\beta}, \quad t \in[0,1] .
$$

Noor [14] has proved that $u \in K_{\beta}$ is a minimum of a differentiable biconvex functions $F$, if and only if, $u \in K_{\beta}$ satisfies the inequality

$$
\left\langle F^{\prime}(u), \beta(v-u)\right\rangle \geq 0, \quad \forall u, v \in K_{\beta}, \quad t \in[0,1]
$$

which is known as the bivariational inequality. For the formulation, applications, numerical methods and other aspects of bivariational inequalities and related optimization problems, see [16, 17, 19] and the references therein.

Definition 6. A strictly positive function $F$ is said to be log-biconvex with respect to an arbitrary bifunction $\beta(.-$.) if

$$
F(u+t \beta(v-u)) \leq(F(u))^{1-t}(F(v))^{-t}, \quad \forall u, v \in K_{\beta}, \quad t \in[0,1] .
$$

We can rewrite the Definition 6 in the following equivalent form as

Definition 7. 13] A strictly positive function $F$ is said to be log-biconvex with respect to an arbitrary bifunction $\beta(.-$.), if

$$
\log F(u+t \beta(v-u)) \leq(1-t) \log F(u)+t \log F(v), \quad \forall u, v \in K_{\beta}, \quad t \in[0,1] .
$$


We use this equivalent (Definition 7) to discuss some new aspects of log-biconvex functions.

If $\log F(u)=e^{f(u)}$, then, we recover the concepts of the exponentially biconvex function, which are mainly due to Noor and Noor [9, 10] as:

Definition 8. 2] A positive function $f$ is said to be exponentially biconvex function, if

$$
e^{f(u+t \beta(v-u))} \leq(1-t) e^{f(u)}+t e^{f(v)}, \quad \forall u, v \in K_{\beta}, \quad t \in[0,1] .
$$

We remark that Definition 8 can be rewritten in the following equivalent way, which is due to Antczak [2].

Definition 9. A function $f$ is said to be exponentially biconvex function, if

$$
f(u+t \beta(v-u)) \leq \log \left[(1-t) e^{f(u)}+t e^{f(v)}\right], \quad \forall u, v \in K_{\beta}, \quad t \in[0,1] .
$$

A function is called the exponentially bincave function $f$, if $-f$ is exponentially biconvex function. For the applications and properties of exponentially biconvex functions, see [9, 10, 16, 17, 19].

We now introduce the concept of strongly log-biconvex functions and study their basic properties.

Definition 10. A strictly positive function $F$ is said to be strongly log-biconvex with respect to an arbitrary bifunction $\beta(.-$.), if there exists a constant $\mu \geq 0$ such that

$$
\begin{array}{r}
\log F(u+t \beta(v-u)) \leq(1-t) \log F(u)+t \log F(v)-\mu t(1-t)\|\beta(v-u)\|^{2}, \\
\forall u, v \in K_{\beta}, \quad t \in[0,1]
\end{array}
$$

Definition 11. A strictly positive function $F$ on the biconvex set $K_{\beta}$ is said to be strongly log-quasi biconvex with respect to an arbitrary bifunction $\beta(.-$.), if

$$
\begin{gathered}
\log F(u+t \beta(v-u)) \leq \max \{\log F(u), \log F(v)\}-\mu t(1-t)\|\beta(v-u)\|^{2}, \\
\forall u, v \in K_{\beta}, t \in[0,1] .
\end{gathered}
$$


Definition 12. A strictly positive function $F$ on the biconvex set $K$ is said to be first kind of strongly log-biconvex with respect to an arbitrary bifunction $\beta(.-$.), if

$$
\begin{gathered}
\log F(u+t \beta(v-u))) \leq\left(\log (F(u))^{1-t}(\log F(v))^{t}-\mu t(1-t)\|\beta(v-u)\|^{2},\right. \\
\forall u, v \in K_{\beta}, t \in[0,1]
\end{gathered}
$$

where $F(\cdot)>0$.

From the above definitions, we have

$$
\begin{aligned}
\log F(u+t \beta(v-u)) & \leq\left(\log (F(u))^{1-t}(\log F(v))^{t}-\mu t(1-t)\|\beta(v-u)\|^{2}\right. \\
& \leq(1-t) \log F(u)+t \log F(v)-\mu t(1-t)\|\beta(v-u)\|^{2} \\
& \leq \max \{\log F(u), \log F(v)\}-\mu t(1-t)\|\beta(v-u)\|^{2}
\end{aligned}
$$

This shows that every fist kind of strongly log-biconvex function is a strongly log-biconvex function and strongly log-biconvex function is a strongly log-quasi-biconvex function. However, the converse is not true.

If $t=1$, then Definitions 2, 12 and 13 , we have:

\section{Condition A.}

$$
\log F(u+\beta(v-u)) \leq F(v), \quad \forall u, v \in K_{\beta}
$$

Condition A plays an important part in the derivation of the main results.

Definition 13. A strictly positive function $F$ is said to be strongly affine log-biconvex function with respect to an arbitrary bifunction $\beta(.-$.), if

$$
\begin{aligned}
\log F(u+t \beta(v-u))= & (1-t) \log F(u)+t \log F(v) \\
& -\mu t(1-t)\|\beta(v-u)\|^{2}, \quad \forall u, v \in K_{\beta}, t \in[0,1] .
\end{aligned}
$$

Let $K_{\beta}=I_{\beta}=[a, a+\beta(b-a)]$ be an interval. We now define the log-biconvex functions on $I_{\beta}$. 
Definition 14. Let $I_{\beta}=[a, a+\beta(b-a)]$. Then $F$ is log-convex function, if and only if,

$$
\left|\begin{array}{ccc}
1 & 1 & 1 \\
a & x & a+\beta(b-a) \\
\log F(a) & \log F(x) & \log F(b)
\end{array}\right| \geq 0 ; \quad a \leq x \leq a+\beta(b-a) .
$$

One can easily show that the following are equivalent:

1. $F$ is a log-biconvex function.

2. $\log F(x) \leq \log F(a)+\frac{\log F(b)-\log F(a)}{\beta(b-a)}(x-a)$.

3. $\frac{\log F(x)-\log F(a)}{x-a} \leq \frac{\log F(b)-\log F(a)}{\beta(b-a)}$.

4. $(a+\eta(b, a)-x) \log F(a)+\beta(a-b) \log F(x)+(x-a) \log F(b)) \geq 0$.

5. $\frac{\log F(a)}{\beta(b-a)(a-x)}+\frac{\log F(x)}{(x-a-\beta(b-a))(a-x)}+\frac{\log F(b)}{\beta(b-a)(x-b)} \leq 0$,

where $x=a+t \beta(b-a) \in[a, a+\beta(b-a)]$.

We also need the following assumption regarding the bifunction $\beta(\cdot-\cdot)$, which play a crucial part in deriving our results.

Condition $\mathbf{C}\left[15\right.$, 16, 17] Let the bifunction $\beta(\cdot-\cdot): K_{\eta} \times K_{\beta} \rightarrow H$ satisfy assumptions

$$
\begin{aligned}
(i) . \quad \beta(\lambda \beta(v-u)) & =\lambda \beta(v-u), \quad \forall u, v \in K_{\beta}, \lambda \in[0,1] \\
(i i) . \quad \beta(v-u-\lambda \beta(v-u)) & =(1-\lambda) \beta(v-u), \quad \forall u, v \in K_{\beta}, \lambda \in[0,1] .
\end{aligned}
$$

Remark 1. Let the bifunction $\beta(.-$.) satisfy the assumption

$$
\beta(v-u)=\beta(v-z)+\beta(z-u), \quad \forall u, v, z \in K_{\beta} .
$$

Clearly, $\beta(v-u)=0, \quad \Leftrightarrow \quad v=u, \quad \forall u, v \in K_{\beta}$ and

$$
\beta(v-u)+\beta(u-v)=0, \quad \forall u, v \in K_{\beta},
$$

that is, the bifunction $\beta(.-$.) is skew symmetric. 


\section{Properties of log-biconvex Functions}

In this section, we consider some basic properties of log-biconvex functions.

Theorem 1. Let $F$ be a strictly log-biconvex function. Then any local minimum of $F$ is a global minimum.

Proof. Let the log-biconvex function $F$ have a local minimum at $u \in K_{\eta}$. Assume the contrary, that is, $F(v)<F(u)$ for some $v \in K$. Since $F$ is a log-biconvex function, so

$\log F(u+t \beta(v-u))<t \log F(v)+(1-t) \log F(u), \quad$ for $\quad 0<t<1$.

Thus

$$
\log F(u+t \beta(v-u))-\log F(u)<t[\log F(v)-\log F(u)]<0,
$$

from which it follows that

$$
\log F(u+t \beta(v-u))<\log F(u)
$$

for arbitrary small $t>0$, contradicting the local minimum.

Theorem 2. If the function $F$ on the biconvex set $K_{\beta}$ is log-biconvex, then the level set

$$
L_{\alpha}=\{u \in K: \log F(u) \leq \alpha, \quad \alpha \in R\}
$$

is a biconvex set.

Proof. Let $u, v \in L_{\alpha}$. Then $\log F(u) \leq \alpha$ and $\log F(v) \leq \alpha$.

Now, $\forall t \in(0,1), \quad w=v+t \beta(u-v) \in K_{\beta}$, since $K_{\beta}$ is an biconvex set. Thus, by the log-biconvexity of $F$, we have

$$
\begin{aligned}
\log F(v+t \beta(u-v)) & \leq(1-t) \log F(v)+t \log F(u) \\
& \leq(1-t) \alpha+t \alpha=\alpha,
\end{aligned}
$$

from which it follows that $v+t \beta(u, v) \in L_{\alpha}$ Hence $L_{\alpha}$ is a biconvex set. 
Theorem 3. A positive function $F$ is a log-biconvex, if and only if

$$
\operatorname{epi}(F)=\{(u, \alpha): u \in K: \log F(u) \leq \alpha, \alpha \in R\}
$$

is a biconvex set.

Proof. Assume that $F$ is log-biconvex function. Let $(u, \alpha), \quad(v, \beta) \in \operatorname{epi}(F)$. Then it follows that $\log F(u) \leq \alpha$ and $\log F(v) \leq \beta$. Thus, $\forall t \in[0,1], \quad u, v \in K_{\beta}$, we have

$$
\begin{aligned}
\log F(u+t \beta(v-u)) & \leq(1-t) \log F(u)+t \log F(v) \\
& \leq(1-t) \alpha+t \beta,
\end{aligned}
$$

which implies that

$$
(u+t \beta(v-u),(1-t) \alpha+t \beta) \in e p i(F) .
$$

Thus epi $(F)$ is an biconvex set. Conversely, let epi $(F)$ be an biconvex set. Let $u, v \in K_{\beta}$. Then $(u, \log F(u)) \in \operatorname{epi}(F)$ and $(v, \log F(v)) \in \operatorname{epi}(F)$. Since epi $(F)$ is a biconvex set, we must have

$$
(u+t \beta(v-u),(1-t) \log F(u)+t \log F(v)) \in e p i(F),
$$

which implies that

$$
\log F(u+t \beta(v-u)) \leq(1-t) \log F(u)+t \log F(u)
$$

This shows that $\mathrm{F}$ is a log-biconvex function.

Theorem 4. A positive function $F$ is quasi log-biconvex, if and only if, the level set

$$
L_{\alpha}=\left\{u \in K_{\beta}, \alpha \in R: \log F(u) \leq \alpha\right\}
$$

is a biconvex set.

Proof. Let $u, v \in L_{\alpha}$. Then $u, v \in K_{\beta}$ and $\max (\log F(u), \log F(v)) \leq \alpha$. 
Now for $t \in(0,1), w=u+t \beta(v-u) \in K_{\beta}$, we have to prove that $u+t \beta(v-u) \in$ $L_{\alpha}$. By the quasi log-biconvexity of $F$, we have

$$
\log F(u+t \beta(v-u)) \leq \max (\log F(u), \log F(v)) \leq \alpha,
$$

which implies that $u+t \beta(v-u) \in L_{\alpha}$, showing that the level set $L_{\alpha}$ is indeed a biconvex set.

Conversely, assume that $L_{\alpha}$ is an biconvex set. Then $\forall u, v \in L_{\alpha}, t \in[0,1]$, $u+t \beta(v-u) \in L_{\alpha}$. Let $u, v \in L_{\alpha}$ for

$$
\alpha=\max (\log F(u), \log F(v) \quad \text { and } \quad \log F(v) \leq \log F(u) .
$$

From the definition of the level set $L_{\alpha}$, it follows that

$$
\log F(u+t \beta(v-u)) \leq \max (\log F(u), \log F(v)) \leq \alpha .
$$

Thus $F$ is a quasi log-biconvex function. This completes the proof.

Theorem 5. Let $F$ be a log-biconvex function. Let $\mu=\inf _{u \in K} F(u)$. Then the set $E=\{u \in K: \log F(u)=\mu\}$ is an biconvex set of $K_{\beta}$. If $F$ is strictly $\log$-convex, then $E$ is a singleton.

Proof. Let $u, v \in E$. For $0<t<1$, let $w=u+t \beta(v-u)$. Since $F$ is a log-biconvex function,

$F(w)=\log F(u+t \beta(v-u)) \leq(1-t) \log F(u)+t \log F(v)=t \mu+(1-t) \mu=\mu$,

which implies that to $w \in E$. and hence $E$ is an biconvex set. For the second part, assume to the contrary that $F(u)=F(v)=\mu$. Since $K_{\beta}$ is a biconvex set, for $0<t<1, u+t \beta(v-u) \in K_{\beta}$. Further, since $F$ is strictly log-biconvex,

$$
\begin{aligned}
\log F(u+t \beta(v-u)) & <(1-t) \log F(u)+t \log F(v) \\
& =(1-t) \mu+t \mu=\mu .
\end{aligned}
$$

This contradicts the fact that $\mu=\inf _{u \in K} F(u)$ and hence the result follows.

Theorem 6. If $F$ is a $\log$-biconvex function such that $\log F(v)<\log F(u), \forall u, v \in$ $K_{\beta}$, then $F$ is a strictly quasi $\log$-biconvex function. 
Proof. By the log-convexity of the function $F, \forall u, v \in K_{\beta}, t \in[0,1]$, we have

$$
\log F(u+t \beta(v-u)) \leq(1-t) \log F(u)+t \log F(v)<\log F(u),
$$

since $\log F(v)<\log F(u)$, which shows that the function $F$ is a strictly quasi log-biconvex function.

\section{Properties of Strongly log-biconvex Functions}

In this section, we discuss some properties of the strongly log-biconvex functions.

Theorem 7. Let $F$ be a differentiable function on the biconvex set $K_{\beta}$ and Condition $C$ hold. Then the function $F$ is log-biconvex function, if and only if

$$
\log F(v)-\log F(u) \geq\left\langle\frac{F^{\prime}(u)}{F(u)}, \beta(v-u)\right\rangle+\mu\|\eta(v, u)\|^{2}, \quad \forall v, u \in K_{\beta} .
$$

Proof. Let $F$ be a strongly log-biconvex function. Then $\log F(u+t \beta(v-u)) \leq(1-t) \log F(u)+t \log F(v)-\mu t(1-t)\|\beta(v-u)\|^{2}, \quad \forall u, v \in K_{\beta}$, which can be written as

$$
\begin{aligned}
\log F(v)-\log F(u) \geq & \left\{\frac{\log F(u+t \beta(v-u))-\log F(u)}{t}\right\} \\
& +\mu(1-t)\|\beta(v-u)\|^{2} .
\end{aligned}
$$

Taking the limit in the above inequality as $t \rightarrow 0$, we have

$$
\log F(v)-\log F(u) \geq\left\langle\frac{F^{\prime}(u)}{F(u)}, \beta(v-u)\right\rangle+\mu\|\beta(v-u)\|^{2},
$$

which is 4.1, the required result.

Conversely, let (4.1) hold. Then $\forall u, v \in K_{\beta}, t \in[0,1], v_{t}=u+t \beta(v-u) \in K_{\beta}$ and using Condition $\mathrm{C}$, we have

$$
\begin{aligned}
\log F(v)-\log F\left(v_{t}\right) \geq & \left.\left\langle\frac{F^{\prime}\left(v_{t}\right)}{F\left(v_{t}\right)}, \beta\left(v-v_{t}\right)\right)\right\rangle+\mu\left\|\beta\left(v-v_{t}\right)\right\|^{2} \\
= & (1-t)\left\langle\frac{F^{\prime}\left(v_{t}\right)}{F\left(v_{t}\right)}, \beta(v-u)\right\rangle \\
& +(1-t)^{2} \mu\|\beta(v-u)\|^{2} .
\end{aligned}
$$


In a similar way, we have

$$
\begin{aligned}
\log F(u)-\log F\left(v_{t}\right) & \geq\left\langle\frac{F^{\prime}\left(v_{t}\right)}{F\left(v_{t}\right)}, \beta\left(u-v_{t}\right)\right\rangle+\mu\left\|\beta\left(u-v_{t}\right)\right\|^{2} \\
& =-t\left\langle\frac{F^{\prime}\left(v_{t}\right)}{F\left(v_{t}\right)}, \beta(v-u)\right\rangle+\mu t^{2}\|\beta(v-u)\|^{2} .
\end{aligned}
$$

Multiplying (4.2) by $t$ and (4.3) by $(1-t)$ and adding the resultant, we have

$$
\begin{array}{r}
\log F(u+t \beta(v-u)) \leq(1-t) \log F(u)+t \log F(v)-\mu t(1-t)\|\beta(v-u)\|^{2}, \\
\forall u, v \in K_{\beta}, t \in[0.1],
\end{array}
$$

showing that $F$ is a strongly log-biconvex function.

Remark 2. From 4.1, we have

$$
F(v) \geq F(u) \exp \left\{\left\langle\frac{F^{\prime}(u)}{F(u)}, \beta(v-u)\right\rangle+\mu\|\beta(v-u)\|^{2}\right\}, \quad \forall u, v \in K_{\beta} .
$$

Changing the role of $u$ and $v$ in the above inequality, we also have

$$
F(u) \geq F(v) \exp \left\{\left\langle\frac{F^{\prime}(v)}{F(v)}, \beta(u-v)\right\rangle+\mu\|\beta(u-v)\|^{2}\right\}, \quad \forall u, v \in K_{\beta} .
$$

Thus, we can obtain the following inequality

$$
\begin{aligned}
F(u)+F(v) \geq & F(v) \exp \left\{\left\langle\frac{F^{\prime}(v)}{F(v)}, \beta(u-v)\right\rangle+\mu\|\beta(u-v)\|^{2}\right\}, \\
& +F(u) \exp \left\{\left\langle\frac{F^{\prime}(u)}{F(u)}, \beta(v-u)\right\rangle+\mu\|\beta(v-u)\|^{2}\right\}, \quad \forall u, v \in K_{\beta} .
\end{aligned}
$$

Theorem 7 enables us to introduce the concept of the log-bimonotone operators, which appears to be a new ones.

Definition 15. The differential $F^{\prime}($.$) is said to be strongly log-bimonotone, if$

$$
\begin{aligned}
& \left\langle\frac{F^{\prime}(u)}{F(u)}, \beta(v-u)\right\rangle+\left\langle\frac{F^{\prime}(v)}{F(v)}, \beta(u-v)\right\rangle \leq \\
& -\mu\left\{\|\beta(v-u)\|^{2}+\|\beta(u-v)\|^{2}\right\}, \quad \forall u, v \in K_{\beta} .
\end{aligned}
$$


Definition 16. The differential $F^{\prime}($.$) is said to be log-bimonotone, if$

$$
\left\langle\frac{F^{\prime}(u)}{F(u)}, \beta(v-u)\right\rangle+\left\langle\frac{F^{\prime}(v)}{F(v)}, \beta(u-v)\right\rangle \leq 0, \quad \forall u, v \in K_{\beta} .
$$

Definition 17. The differential $F^{\prime}($.$) is said to be log-pseudo-bimonotone, if$

$$
\left\langle\frac{F^{\prime}(u)}{F(u)}, \beta(v-u)\right\rangle \geq 0, \quad \Rightarrow-\left\langle\frac{F^{\prime}(v)}{F(v)}, \beta(u-v)\right\rangle \geq 0, \quad \forall u, v \in K_{\beta} .
$$

From these definitions, it follows that strongly log-bimonotonicity implies log-bimonotonicity implies log-pseudo-bimonotonicity, but the converse is not true.

Theorem 8. Let $F$ be differentiable strongly log-biconvex function on the biconvex set $K_{\beta}$. Let Condition $C$ and Condition A hold. Then (4.1) holds, if and only if, $F^{\prime}($.$) satisfies$

$$
\begin{aligned}
& \left\langle\frac{F^{\prime}(u)}{F(u)}, \beta(v-u)\right\rangle+\left\langle\frac{F^{\prime}(v)}{F(v)}, \beta(u-v)\right\rangle \\
\leq & -\mu\left\{\|\beta(v-u)\|^{2}+\|\beta(u-v)\|^{2}\right\}, \quad \forall u, v \in K_{\beta} .
\end{aligned}
$$

Proof. Let $F$ be a strongly log-biconvex function on the biconvex set $K_{\beta}$. Then, from Theorem 7 , we have

$$
\log F(v)-\log F(u) \geq\left\langle\frac{F^{\prime}(u)}{F(u)}, \beta(v-u)\right\rangle+\mu\|\beta(v-u)\|^{2}, \quad \forall u, v \in K_{\beta} .
$$

Changing the role of $u$ and $v$ in 4.5 , we have

$$
\log F(u)-\log F(v) \geq\left\langle\frac{F^{\prime}(v)}{F(v)}, \beta(u-v)\right\rangle+\|\beta(u-v)\|^{2}, \quad \forall u, v \in K_{\beta} .
$$

Adding (4.5) and 4.6), we have

$$
\begin{aligned}
& \left\langle\frac{F^{\prime}(u)}{F(u)}, \beta(v-u)\right\rangle+\left\langle\frac{F^{\prime}(v)}{F(v)}, \beta(u-v)\right\rangle \\
\leq & -\mu\left\{\|\beta(v-u)\|^{2}+\|\beta(u-v)\|^{2}\right\}, \quad \forall u, v \in K_{\beta}
\end{aligned}
$$

which shows that $F^{\prime}$ is a strongly log-monotone. 
Conversely, from (4.4) and Condition C, we have

$$
\begin{aligned}
& \left\langle\frac{F^{\prime}(v)}{F(v)}, \beta(u-v)\right\rangle \\
\leq & -\mu\left\{\|\beta(v-u)\|^{2}+\|\eta(u, v)\|^{2}\right\}-\left\langle\frac{F^{\prime}(u)}{F(u)}, \beta(v-u)\right\rangle, \quad \forall u, v \in K_{\beta} .
\end{aligned}
$$

Since $K$ is an convex set, $\forall u, v \in K_{\beta}, \quad t \in[0,1] v_{t}=u+t \beta(v-u) \in K_{\beta}$.

Taking $v=v_{t}$ in (4.7), we have

$$
\begin{aligned}
& \left\langle\frac{F^{\prime}\left(v_{t}\right)}{F\left(v_{t}\right)}, \beta\left(u-v_{t}\right)\right\rangle \\
\leq & -\mu\left\{\left\|\beta\left(v_{t}-u\right)\right\|^{2}+\left\|\beta\left(u-v_{t}\right)\right\|^{2}\right\}-\left\langle\frac{F^{\prime}(u)}{F(u)}, \beta\left(v_{t}-u\right)\right\rangle, \quad \forall u, v \in K_{\beta} .
\end{aligned}
$$

Using Condition C, we obtain

$$
\left.\left\langle\frac{F^{\prime}\left(v_{t}\right)}{F\left(v_{t}\right)}, \beta(v-u)\right\rangle \geq\left\langle\frac{F^{\prime}(u)}{F(u)}, \beta(v-u)\right\rangle+2 \mu t\|\beta(v-u)\|^{2}\right\rangle .
$$

Consider the auxiliary function

$$
\xi(t)=\log F(u+t \beta(v-u)), \quad \forall u, v \in K_{\beta},
$$

from which, we have

$$
\xi(1)=\log F(u+\beta(v-u)), \quad \xi(0)=\log F(u) .
$$

Then, from 4.8), we have

$$
\xi^{\prime}(t)=\left\langle\frac{F^{\prime}\left(v_{t}\right)}{F\left(v_{t}\right)}, \beta(v-u)\right\rangle \geq\left\langle\frac{F^{\prime}(u)}{F(u)}, \beta(v-u)\right\rangle+2 \mu t\|\beta(v-u)\|^{2} .
$$

Integrating 4.9 between 0 and 1 , we have

$$
\xi(1)-\xi(0)=\int_{0}^{1} \xi^{\prime}(t) d t \geq\left\langle\frac{F^{\prime}(u)}{F(u)}, \beta(v-u)\right\rangle+\mu\|\beta(v-u)\|^{2} .
$$

Thus it follows, using Condition A, that

$$
\log F(v)-\log F(u) \geq\left\langle\frac{F^{\prime}(u)}{F(u)}, \beta(v-u)\right\rangle+\mu\|\beta(v-u)\|^{2},
$$

which is the required 4.1. 
We now give a necessary condition for log-pseudo biconvex function.

Theorem 9. Let $F^{\prime}($.$) be a log-pseudo bimonotone and let Condition C$ and Condition $A$ hold. Then $F$ is a log-pseudo biconvex function.

Proof. Let $F^{\prime}($.$) be a log-pseudo bimonotone. Then,$

$$
\left\langle\frac{F^{\prime}(u)}{F(u)}, \beta(v-u)\right\rangle \geq 0, \quad \forall u, v \in K_{\beta},
$$

implies that

$$
-\left\langle\frac{F^{\prime}(v)}{F(v)}, \beta(v-u)\right\rangle \geq 0
$$

Since $K_{\eta}$ is an biconvex set, $\forall u, v \in K_{\beta}, t \in[0,1], v_{t}=u+t \beta(v-u) \in K_{\beta}$.

Taking $v=v_{t}$ in 4.10 and using Condition $\mathrm{C}$, we have

$$
\left\langle\frac{F\left(v_{t}\right)}{F^{\prime}\left(v_{t}\right)}, \beta(v-u)\right\rangle \geq 0 .
$$

Consider the auxiliary function

$$
\xi(t)=\log F(u+t \beta(v-u))=\log F\left(v_{t}\right), \quad \forall u, v \in K_{\beta}, t \in[0,1],
$$

which is a differentiable function. Then, using 4.11, we have

$$
\xi^{\prime}(t)=\left\langle\frac{F^{\prime}\left(v_{t}\right)}{F\left(v_{t}\right)}, \beta(v-u)\right\rangle \geq 0 .
$$

Integrating the above relation between 0 to 1 , we have

$$
\xi(1)-\xi(0)=\int_{0}^{1} \xi^{\prime}(t) d t \geq 0 .
$$

Using Condition A, we have

$$
\log F(v)-\log F(u) \geq 0,
$$

showing that $F$ is a log-pseudo biconvex function. 
Definition 18. The function $F$ is said to be sharply $\log$-pseudo biconvex, if there exists a constant $\mu>0$ such that

$$
\begin{aligned}
\left\langle\frac{F^{\prime}(u)}{F(u)}, \beta(v-u)\right\rangle & \geq 0 \\
& \Rightarrow \\
\log F(v) & \geq \log F(u+t \beta(v-u)), \quad \forall u, v \in K_{\beta}, t \in[0,1] .
\end{aligned}
$$

Theorem 10. Let $F$ be a sharply log-pseudo biconvex function on the biconvex set $K_{\beta}$. Then

$$
\left\langle\frac{F^{\prime}(v)}{F(v)}, \beta(v-u)\right\rangle \geq 0, \quad \forall u, v \in K_{\beta} .
$$

Proof. Let $F$ be a sharply log-pseudo biconvex function on $K_{\beta}$. Then

$$
\log F(v) \geq \log F(v+t \beta(u-v)), \quad \forall u, v \in K_{\beta}, t \in[0,1] .
$$

from which we have

$$
0 \leq \lim _{t \rightarrow 0}\left\{\frac{\log F(v+t \beta(u-v))-\log F(v)}{t}\right\}=\left\langle\frac{F^{\prime}(v)}{F(v)}, \beta(v-u)\right\rangle,
$$

the required result.

Definition 19. A function $F$ is said to be a log-pseudo biconvex function with respect to a strictly positive bifunction $B(.,$.$) , such that$

$$
\begin{aligned}
\log F(v) & <\log F(u) \\
& \Rightarrow \\
\log F(u+t \beta(v-u)) & <\log F(u)+t(t-1) B(v, u), \forall u, v \in K_{\beta}, t \in[0,1] .
\end{aligned}
$$

Theorem 11. If the function $F$ is strongly log-biconvex function such that $\log F(v)<\log F(u)$, then the function $F$ is a strongly log-pseudo biconvex. 
Proof. Since $\log F(v)<\log F(u)$ and $F$ is strongly log-convex function, then $\forall u, v \in K_{\eta}, \quad t \in[0,1]$, we have

$$
\begin{aligned}
& \log F(u+t \beta(v-u)) \\
\leq & \log F(u)+t(\log F(v)-\log F(u))-\mu t(1-t)\|\beta(v-u)\|^{2} \\
< & \log F(u)+t(1-t)(\log F(v)-\log F(u))-\mu t(1-t)\|\beta(v-u)\|^{2} \\
= & \log F(u)+t(t-1)(\log F(u)-\log F(v))-\mu t(1-t)\|\beta(v-u)\|^{2} \\
< & \log F(u)+t(t-1) B(u, v)-\mu t(1-t)\|\beta(v-u)\|^{2},
\end{aligned}
$$

where $B(u, v)=\log F(u)-\log F(v)>0$. This shows that the function $F$ is strongly log-biconvex function.

We now show that the difference of strongly log-biconvex function and affine strongly log-biconvex function is again a log-biconvex function.

Theorem 12. Let $f$ be a affine strongly log-biconvex function. Then $F$ is a strongly log-biconvex function, if and only if, $g=F-f$ is a log-biconvex function. Proof. Let $f$ be an affine strongly log-biconvex function. Then

$$
\begin{aligned}
\log f((u+t \beta(v-u))= & (1-t) \log f(u)+t \log f(v) \\
& -\mu t(1-t)\|\beta(v-u)\|^{2}, \forall u, v \in K_{\beta}, t \in[0,1](
\end{aligned}
$$

From the strongly log-biconvexity of $F$, we have

$$
\begin{aligned}
\log F(u+t \beta(v-u)) \leq & (1-t) \log F(u)+t \log F(v) \\
& -\mu t(1-t)\|\beta(v-u)\|^{2}, \forall u, v \in K_{\beta}, t \in[0,1] .
\end{aligned}
$$

From 4.12 ) and (4.13), we have

$$
\begin{aligned}
\log F((u+t \beta(v-u))-\log f((u+t \beta(v-u)) \leq & (1-t)(\log F(u)-\log f(u)) \\
& +t(\log F(v)-\log f(v))(4.14)
\end{aligned}
$$

from which it follows that

$$
\begin{aligned}
\log g((u+t \beta(v-u)) & =\log F((u+t \beta(v-u)-\log f((u+t \beta(v-u)) \\
& \leq(1-t)(\log F(u)-\log f(u))+t(\log F(v)-\log f(v)) .
\end{aligned}
$$


This shows that $g=F-f$ is a log-biconvex function.

The inverse implication is obvious.

We remark that, if a strictly positive function $F$ is a strongly log-biconvex function, then we have

$$
\begin{aligned}
& \log F(u+t \beta(v-u))+\log F(v+t \beta(u-v)) \\
\leq & \log F(u)+\log F(v)-2 \mu t(1-t)\|\beta(v-u)\|^{2}, \forall u, v \in K_{\beta}, t \in[0,1],
\end{aligned}
$$

which is called the Wright strongly log-biconvex function.

From 4.15, we have

$$
\begin{aligned}
& \log F(u+t \eta(v, u)) F(v+t \beta(u-v)) \\
= & \log F(u+t \beta(v-u))+\log F(v+t \beta(u-v)) \\
\leq & \log F(u)+\log F(v) \\
= & \log F(u) F(v), \quad \forall u, v \in K_{\beta}, t \in[0,1] .
\end{aligned}
$$

This implies that

$$
F\left((u+t \beta(v-u)) F(t u+(1-t) v) \leq F(u) F(v), \quad \forall u, v \in K_{\beta}, t \in[0,1],\right.
$$

which shows that a strictly positive function $F$ is a multiplicative Wright strongly log-biconvex function. It is an interesting problem to study the properties and applications of the Wright log-biconvex functions.

\section{$5 \quad$ Parallelogram Laws}

In this section, we derive some new parallelogram laws for uniformly Banach spaces as a novel application of strongly log-biconvex functions.

From Definition 13, we have

$$
\begin{aligned}
\log F(u+t \beta(v-u))= & (1-t) \log F(u)+t \log F(v) \\
& -\mu t(1-t)\|\beta(v-u)\|^{2}, \forall u, v \in K_{\beta, t} \in[0,1] .
\end{aligned}
$$


Taking $t=\frac{1}{2}$ in 5.1 , we have

$$
\begin{aligned}
& \log F\left(u+\frac{1}{2} \beta(v-u)\right)+\mu \frac{1}{2^{2}}\|\beta(v-u)\|^{2} \\
= & \frac{1}{2}\{\log F(u)+\log F(v)\}, \forall u, v \in K_{\beta},
\end{aligned}
$$

which is called the log parallelogram law for the Banach spaces involving strongly log-biconvex functions.

(I). If $\log F(u)=\|u\|^{2}$, then equation (5.2) can be written as

$$
\left.\| 2 u+\frac{1}{2} \beta(v-u)\right)\left\|^{2}+\mu\right\| \beta(v-u) \|^{2}=2\left\{\|u\|^{2}+\|v\|^{2}\right\}, \forall u, v \in K_{\beta} .
$$

(II). If $\beta(v, u)=v-u$, then (5.3) reduces to the parallelogram-like law as:

$$
\|v+u\|^{2}+\mu\|v-u\|^{2}=2\left\{\|u\|^{2}+\|v\|^{2}\right\},
$$

which is the well known parallelogram law, which characterizes the inner product spaces.

The log-parallelogram law 5.2 characterizes the inner product spaces involving exponentially biconvex function. Also, see [6, 20] for the derivation and other properties of the inner product spaces.

For suitable and appropriate choice of the function $\beta(.-$.), one can obtain a wide class of new parallelogram laws, which can be used to characterize various classes of inner products.

\section{Optimization}

We now discuss the optimality condition for the differentiable strongly log-biconvex functions, which is the main motivation of our next result.

Theorem 13. Let $F$ be a differentiable strongly log-biconvex function. If $u \in K_{\beta}$ is a minimum of the function $F$, then $u \in K_{\beta}$ satisfies the inequality

$$
\log F(v)-\log F(u) \geq \mu\|\eta(v, u)\|^{2}, \forall u, v \in K_{\beta} .
$$


Proof. Let $u \in K_{\beta}$ be a minimum of the function $F$. Then

$$
F(u) \leq F(v), \forall v \in K_{\beta} .
$$

from which, we have

$$
\log F(u) \leq \log F(v), \forall v \in K_{\beta} .
$$

Since $K$ is a biconvex set, so, $\forall u, v \in K_{\beta}, \quad t \in[0,1], v_{t}=u+t \beta(v-u) \in K_{\beta}$.

Taking $v=v_{t}$ in 6.2 , we have

$$
0 \leq \lim _{t \rightarrow 0}\left\{\frac{\log F(u+t \beta(v-u))-\log F(u)}{t}\right\}=\left\langle\frac{F^{\prime}(u)}{F(u)}, \beta(v-u)\right\rangle .
$$

Since $F$ is differentiable strongly log-biconvex function, so

$$
\begin{aligned}
\log F(u+t \beta(v-u)) \leq & \log F(u)+t(\log F(v)-\log F(u)) \\
& -\mu t(1-t)\|\beta(v-u)\|^{2}, \forall u, v \in K_{\beta}, t \in[0,1] .
\end{aligned}
$$

Using 6.3, we have

$$
\begin{aligned}
\log F(v)-\log F(u) & \geq \lim _{t \rightarrow 0}\left\{\frac{\log F(u+t \beta(v-u))-\log F(u)}{t}\right\}+\mu\|\beta(v-u)\|^{2} \\
& =\left\langle\frac{F^{\prime}(u)}{F(u)}, \beta(v-u)\right\rangle+\mu\|\beta(v-u)\|^{2} \geq 0 .
\end{aligned}
$$

Thus, it follows that

$$
\log F(v)-\log F(u) \geq \mu\|\beta(v-u)\|^{2},
$$

which is the required result 6.1.

Remark 3. We note that, if $u \in K_{\beta}$ satisfies the

$$
\left\langle\frac{F^{\prime}(u)}{F(u)}, \beta(v-u)\right\rangle+\mu\|\beta(v-u)\|^{2} \geq 0, \forall v \in K_{\beta},
$$

then $u \in K_{\beta}$ is a minimum of a strongly log-biconvex function $F$. The inequality of the type (6.4) is called the log-bivariational inequality and appears to be a new one. It is an interesting problem to study the existences of a solution of the log-bivariational inequalities and develop some numerical methods. For the applications, formulations, numerical methods and other aspects of variational inequalities, see [7, 8, 15, 16, 17, 19, 20, 24] and the references therein 


\section{Conclusion}

Several new classes of strongly log-biconvex functions have been introduced and their properties are investigated. It has been shown that log-biconvex functions enjoy several properties which convex functions have. Some parallelogram laws are obtained as applications of the strongly log-biconvex functions, which can be used to characterize the inner product spaces. We have shown that the minimum of the differentiable strongly log-convex functions can be characterized by a new class of variational inequalities, which is called the log-bivariational inequality. One can explore the applications of the log-bivariational inequalities in pure and applied sciences. This may stimulate further research.

\section{Acknowledgements}

The authors wish to express our deepest gratitude to our teachers, colleagues, collaborators and friends, who have direct or indirect contributions in the process of this paper.

\section{References}

[1] G. Alirezaei and R. Mazhar, On exponentially concave functions and their impact in information theory, J. Inform. Theory Appl. 9(5) (2018), 265-274.

[2] T. Antczak, On $(p, r)$-invex sets and functions, J. Math. Anal. Appl. 263 (2001), 355-379. https://doi.org/10.1006/jmaa.2001.7574

[3] S. N. Bernstein, Sur les fonctions absolument monotones, Acta Math. 52 (1929), 1-66. https://doi.org/10.1007/BF02592679

[4] B. B. Mohsen, M. A. Noor, K. I. Noor and M. Postolache, Strongly convex functions of higher order involving bifunction, Mathematics 7(11) (2019), 1028. https://doi.org/10.3390/math7111028

[5] C. P. Niculescu and L. E. Persson, Convex Functions and Their Applications, Springer-Verlag, New York, 2018. 
[6] K. Nikodem and Z. S. Pales, Characterizations of inner product spaces by strongly convex functions, Banach J. Math. Anal. 1 (2011), 83-87.

https://doi.org/10.15352/bjma/1313362982

[7] M. A. Noor, New approximation schemes for general variational inequalities, $J$. Math. Anal. Appl. 251 (2000), 217-229.

https://doi.org/10.1006/jmaa.2000.7042

[8] M. A. Noor, Some developments in general variational inequalities, Appl. Math. Comput. 152 (2004), 199-277. https://doi.org/10.1016/S0096-3003(03)00558-7

[9] M. A. Noor and K. I. Noor, Exponentially convex functions, J. Orisa Math. Soc. 38(01-02) (2019), 33-51.

[10] M. A. Noor and K. I. Noor, Strongly exponentially convex functions, U.P.B. Bull. Sci. Appl. Math. Series A 81(4) (2019), 75-84.

[11] M. A. Noor and K. I. Noor, New classes of strongly exponentially preinvex functions, AIMS Math. 4(6) (2019), 1554-1568. https://doi.org/10.3934/math.2019.6.1554

[12] M. A. Noor and K. I. Noor, Strongly log-convex functions, Inform. Sci. Letters 10(1) (2021), 33-38. https://doi.org/10.18576/isl/100105

[13] M. A. Noor and K. I. Noor, New characterizations of higher order strongly log-convex functions, J. Adanc. Math. Studies 14(3) (2021).

[14] M. A. Noor and K. I. Noor, Higher order strongly generalized convex functions, Appl. Math. Inf. Sci. 14(1) (2020), 133-139. https://doi.org/10.18576/amis/140117

[15] M. A. Noor and K. I. Noor, Higher order strongly exponentially biconvex functions and bivariational inequalities, J. Math. Anal. 12(2) (2021), 23-43.

[16] M. A. Noor and K. I. Noor, Higher order strongly biconvex functions and biequilibrium problems, Advan. Lin. Algebr. Matrix Theory 11(2) (2021).

[17] M. A. Noor and K. I. Noor, Exponentially biconvex functions and bivariational inequalities, in: Advances in Mathematical Analysis and Multidisciplinary Applications (Edited: B. Hazarika, S. Acharjee and H. M. Srivastava), CRC Press, Year. 
[18] M. A. Noor, K. I. Noor and M. U. Awan, New prospective of log-convex functions, Appl. Math. Inform. Sci. 14(5) (2021), 847-854. https://doi.org/10.18576/amis/140512

[19] M. A. Noor, K. I. Noor and M. T. Rassias, Strongly biconvex functions and bivariational inequalities, in:Mathematical Analysis, Optimization, Approximation and Applications (Edited: Panos M. Pardalos and Th. M. Rassias), World Scientific Publishing Company, Singapore, Year.

[20] M. A. Noor, K. I. Noor and M. Th. Rassias, New trends in general variational inequalities, Acta Appl. Math. 170(1) (2020), 981-1064.

https://doi.org/10.1007/s10440-020-00366-2

[21] S. Pal and T. K. Wong, Exponentially concave functions and a new information geometry, Ann. Probab. 46(2) (2018), 1070-1113.

https://doi.org/10.1214/17-AOP1201

[22] B. T. Polyak, Existence theorems and convergence of minimizing sequences in extremum problems with restrictions, Soviet Math. Dokl. 7 (1966), 2-75.

[23] Y. X. Zhao, S. Y. Wang and L. Coladas Uria, Characterizations of $r$-convex functions, J. Optim. Theory Appl. 145 (2010), 186-195.

https://doi.org/10.1007/s10957-009-9617-1

[24] D. L. Zhu and P. Marcotte, Co-coercivity and its role in the convergence of iterative schemes for solving variational inequalities, SIAM J. Optim. 6(3) (1996), 714-726. https://doi.org/10.1137/S1052623494250415

This is an open access article distributed under the terms of the Creative Commons Attribution License (http://creativecommons.org/licenses/by/4.0/), which permits unrestricted, use, distribution and reproduction in any medium, or format for any purpose, even commercially provided the work is properly cited. 